\title{
La « ville durable »
}

Personnages en quête d'auteur

"Sustainable City" - Characters in Search of an Author

\section{Luna D 'Emilio}

\section{(2) OpenEdition}

\section{Journals}

Édition électronique

URL : http://journals.openedition.org/crau/552

DOI : 10.4000/crau.552

ISSN : 2547-5746

Éditeur

Éditions du patrimoine

\section{Édition imprimée}

Date de publication : 1 novembre 2012

Pagination : $97-102$

ISBN : 978-2-7577-0108-9

ISSN : 1296-4077

Référence électronique

Luna D 'Emilio, "La « ville durable » », Les Cahiers de la recherche architecturale et urbaine [En ligne], 26/27 | 2012, mis en ligne le 01 novembre 2017, consulté le 01 mai 2019. URL : http:// journals.openedition.org/crau/552; DOI : 10.4000/crau.552 
La diffusion de la notion de ville durable conduit à s'interroger sur son contenu scientifique et sur son rapport avec la réflexion sur l'urbain. Sont questionnés ici le statut et les particularités de la recherche urbaine et des disciplines qui la nourrissent ainsi que les possibles postures intellectuelles qui en dérivent. Cet article développe une réflexion sur les limites et les statuts disciplinaires, à partir de deux niveaux : celui de la légitimation sur le plan des cultures disciplinaires, et celui de l'instrumentalisation sur le plan des pratiques de transformation de l'espace. L'hypothèse est que le questionnement épistémologique est une condition pour sonder la portée et les limites de la notion de ville durable, et pour mettre en perspective son rapport émergent avec les disciplines de I'architecture et de l'urbanisme.

\section{La « ville durable »}

Personnages en quête d'auteur ${ }^{1}$

LUNA D'EMILIO

Le débat actuel autour de la «ville durable » est un débat politique, technique et culturel. II fait référence à la question de la transformation de l'espace, des temporalités urbaines, en essayant de repenser le lien entre urbs et civitas. Malgré cette posture et ces enjeux, le débat laisse persister beaucoup d'ambiguïtés et d'incertitudes. D'une part, si le mot « durable » est de plus en plus présent dans la production scientifique liée à la recherche urbaine, d'autre part, les contributions qui la concernent sont hétérogènes aussi bien sur le type de discours que sur les échelles de référence et les objectifs ; il s'agit en fait d'un concept pas encore consolidé, qui rencontre aussi des inerties et des méfiances ${ }^{2}$. La diffusion du concept de ville durable nous conduit à nous interroger sur son contenu scientifique et sur son rapport avec la réflexion sur l'urbain qui l'a précédée. Nous essayerons dans cet écrit de proposer une contribution dans ce sens. Nous questionnerons le statut et les particularités de la recherche urbaine et des disciplines qui la nourrissent ainsi que les possibles postures intellectuelles qui en dérivent. Notre

\footnotetext{
1. Six personnages en quête d'auteur, la pièce théâtrale de l'écrivain et dramaturge italien Luigi Pirandello, nous a inspiré ce titre car le personnage est vu en tant que dispositif capable de nourrir une réflexion sur le rapport entre réalité et idées. Cet article se situe dans le contexte de la thèse $L a$ ville durable en projet. Entre réflexion et praxis, continuité et rupture : le cas de Strasbourg (2009 - en cours). Cette thèse est en co-tutelle entre
}

I'université de Florence (département nationale supérieure de Strasbourg (Amup, C. Mazzoni).

2. Voir par exemple le mouvement dit de la " décroissance», qui s'est développé autour des théories de I'économiste Nicholas GeorgescuRoegen. d'urbanisme, M. Morandi) et l'école 
hypothèse est que le questionnement épistémologique est une condition pour sonder la portée et les limites de la notion de ville durable, et pour mettre en perspective son rapport émergent avec les disciplines de l'architecture et de l'urbanisme.

\section{Sur la recherche urbaine en tant que science : objets, sujets, itinéraires Des objets aux sujets}

Dans la réflexion du chercheur, les questions liées au sens de sa propre activité sont centrales. Le questionnement épistémologique se pose tout le long du processus de recherche : il en définit l'orientation et il en nourrit les réflexions. Dans l'analyse urbaine, si un premier type de discours se focalise sur la question des objets de recherche et essaye d'en saisir les caractères, un deuxième type de discours aborde les relations possibles entre les acteurs de la recherche urbaine et ces mêmes objets. On sait aussi que la recherche urbaine est animée par un double objectif: I'un de type normatif, l'autre de type cognitif ${ }^{3}$. La recherche normative veut déterminer les actions nécessaires à accomplir sur un objet matériel (la ville), tandis que la recherche cognitive veut produire de la connaissance en rapport avec cet objet, indépendamment de ses applications pratiques possibles. Ces deux approches sont à l'origine de deux visions différentes du territoire : celle des techniciens et celle des savants, parfois antinomiques ${ }^{4}$.

Il est vrai que d'un point de vue cognitif, la ville - objet empirique - est associée à plusieurs objets scientifiques issus de différentes disciplines tels que l'urbanisme, la sociologie ou l'économie. Chaque discipline donne une représentation cognitive de l'objet empirique définissant de facto un objet scientifique associé ${ }^{5}$. Dans ce contexte, la recherche se pose comme une action capable de redéfinir en continu les divers objets scientifiques. D'ailleurs, quand des nouveaux concepts sont intégrés dans les disciplines, ils modifient les représentations cognitives de l'objet empirique. II en ressort que les objets scientifiques sont instables, résultats d'un travail de redéfinition permanente.

Ce type de discours introduit la notion du sujet dans lequel a lieu la représentation de l'objet empirique. On se pose donc la question du rapport entre objet et sujet, de la définition et du rôle de celui-ci. L'approche positiviste considérait le sujet en tant que tabula rasa, capable de parvenir à une observation objective et " neutre » de la réalité, issue de l'application d'une méthode définie à priori. La réflexion épistémologique a changé cette vision : les frontières entre sujet et objet deviennent plus floues, et les deux s'influencent réciproquement. Ce changement porte à abandonner l'idée que la recherche doit " trouver » quelque chose, elle doit plutôt « construire » quelque chose.

Parfois, le processus de recherche exige de construire des nouvelles catégories conceptuelles pour répondre aux questions et aux problèmes posés. Cet ensemble d'opérations demande au sujet de sortir de son champ disciplinaire. En particulier, le caractère multidimensionnel de la ville demande une approche inter et transdisciplinaire ${ }^{6}$. Par conséquent, le sujet est un élément actif de la recherche, porteur d'un bagage culturel capable de l'influencer. Cela montre l'impor-
3. Ce dualisme est récurrent dans le champ de la recherche urbaine. Voir Françoise Choay, La règle et le modèle, Paris, Seuil, 1980 ; Pier Giorgio Gerosa, Éléments pour une histoire des théories sur la ville comme artefact et forme spatiale (XVIII $-X X^{e}$ siècles), Strasbourg, université de sciences humaines, 1992 ; Albert Lévy, Vittorio Spigai, II piano e I'architettura della città, Venise, Cluva, 1989

4. André Corboz, "La description : entre lecture et écriture », Le Territoire comme palimpseste, Besançon, Les Éditions de I'Imprimeur, 2001, p. 249-258; paru dans Faces, $n^{\circ} 48$, automne 2000 , p. 52-54.

5. Philippe Boudon, «Discipline, métadiscipline ", Cahiers thématiques $n^{\circ} 1$, Lille, École nationale supérieure d'architecture et de paysage, 2001, p. 14-25

6. Dans une approche interdisciplinaire, les disciplines prennent part à un projet collectif. Elles contribuent avec leurs savoirs et méthodes, tout en gardant leur spécificité disciplinaire ; dans une approche transdisciplinaire un véritable dépassement des frontières disciplinaires a lieu, et on constate la création ou l'acquisition de compétences communes. Voir Edgar Morin, "Sur l'interdisciplinarité », Bulletin Interactif du Centre International de Recherches et Études transdisciplinaires $n^{\circ} 2,1994$. Consultable en ligne : http://basarab. nicolescu.perso.sfr.fr/ciret/bulletin/b2c2. htm. 
tance des différents acteurs impliqués, chacun porteur d'un niveau d'expérience et de lecture particulier. La recherche urbaine se pose donc au croisement de ces différentes représentations.

\section{Les itinéraires possibles : quatre personnages}

Nous essayerons, par une métaphore empruntée à André $\mathrm{Corboz}^{7}$, de faire une synthèse de ce qu'on vient d'exposer. II s'agit de comprendre les actions possibles de la recherche à l'aide d'un espace abstrait quadripartite généré par deux axes. Ceux-ci symbolisent deux aspects indépendants mais complémentaires de la recherche : les frontières disciplinaires d'une part, et la relation sujet-objet, d'autre part. Cette métaphore voit l'introduction de quatre personnages.

À ce propos, Corboz nous parle de deux groupes d'acteurs dans le champ du savoir scientifique : les premiers - définis " prétoriens de l'orthodoxie »-insistent sur l'autonomie disciplinaire ; les deuxièmes - « aventuriers sans foi ni religion » - franchissent n'importe quelle séparation entre les champs du savoir. Ces positions sont stériles, puisque les premiers n'acceptent pas la critique de la méthode disciplinaire en tant qu'outil d'exploration de la complexité ; de leur coté, les aventuriers ne se posent guère la question de la méthode.

À ces deux extrêmes, Corboz ajoute deux autres figures pittoresques, définies à partir des rapports possibles entre sujet et objet qu'on vient d'exposer. D'un côté, les "notaires", de dérivation positiviste, croient que la réalité peut être enregistrée par un observateur parfaitement neutre; de l'autre, les "ventriloques", de dérivation idéaliste, pensent que la réalité dérive uniquement de leur activité de recherche. Ces deux cas représentent, de leur coté aussi des positions extrêmes, étant donné que les premiers ont tendance à nier le sujet, les seconds à nier l'objet.

Ces quatre figures extrêmes - prétoriens, aventuriers, notaires et ventriloques - sont utiles puisqu'elles aident à comprendre les limites des différentes postures intellectuelles : ils contribuent à délimiter un véritable champ d'action de la recherche. D'infinies possibilités méthodologiques et épistémologiques peuvent avoir lieu entre ces extrêmes; c'est à chaque chercheur de trouver sa propre place.

\section{Sur la "ville durable» Un concept central dans la réflexion contemporaine}

Comme exposé auparavant, la ville durable, concept polysémique dont le contenu n'a pas été défini de manière univoque, se trouve depuis une dizaine d'années de plus en plus au centre de débats. En particulier, même si l'on constate une effervescence du concept sur plusieurs échelles et dans des contextes divers, il est opportun de mettre en question sa validité et sa légitimité en tant qu'objet de recherche.

$\mathrm{Si}$ on s'interroge sur le rapport de la ville durable avec la recherche issue de différentes disciplines, plusieurs questions se posent. S'agit-il d'une discontinuité dans l'histoire de la pensée scientifique qui fait référence à la ville ${ }^{8}$ ? Cette discontinuité ouvre-t-elle la voie à une approche transdisciplinaire du concept ? Comment garder une approche omni-compréhensive face à la prolifération des apports spécifiques? Comme
Voir Gérard Baudin, Philippe Genestier, «La ville : objet de connaissance, objet de discours, objet d'action?", Les Annales de la recherche urbaine $n^{\circ} 104,2008$, p. 175-181.

7. A. Corboz, «La recherche : trois apologues ", dans Roger Durand (éd.), C'est la faute à Voltaire, c'est la faute à Rousseau. Recueil anniversaire pour Jean-Daniel Candaux, Genève, Droz coll. «Varia », 1997.
8. Nicole Mathieu, Yves Guermond (dir.), La ville durable, du politique au scientifique, Paris, Cemagref Éditions, 2005. 
on l'a souligné auparavant, c'est la représentation d'une discipline penchée sur un objet empirique qui donne lieu à un objet scientifique. Alors comment la ville durable peut se constituer en tant qu'objet scientifique sans dériver d'aucune discipline en particulier?

On relève les réflexions d'ordre politique, d'une part, et celles d'ordre strictement technique, d'autre part. Pour certains, la durabilité urbaine est vue en tant que concept général d'orientation, pour d'autres elle n'est qu'un outil opératif. Nous croyons que cette ambiguïté dérive de la nature de l'objet-ville durable, résultat de l'application d'une représentation politique (le développement durable) de la ville en tant qu'objet empirique ; le questionnement épistémologique sur la ville durable peut aider à dépasser cette dualité. Une réflexion sur les limites et les statuts disciplinaires s'impose, de nouveau?. Nous allons la développer à partir de deux niveaux : celui de la légitimation sur le plan des cultures disciplinaires, et celui de l'instrumentalisation sur le plan des pratiques de transformation de l'espace. Les catégories conceptuelles de cette opération seraient celles de la continuité et de la rupture, en rapport avec les discours sur la ville qui l'ont précédée.

\section{De l'histoire aux cultures disciplinaires : légitimation}

Bien que la ville durable semble être le résultat d'une réflexion née en dehors de l'urbanisme ${ }^{10}$, pour certains ${ }^{11}$, la diffusion du concept de développement durable est à l'origine d'un véritable tournant de la culture urbanistique. Mais cette transformation serait de nature incrémentale, caractérisée par l'introduction d'un concept extérieur à la recherche urbaine, qui se façonne par approximations successives, résistances et méfiances. Dans cette perspective la question des cultures des différents acteurs urbains devient centrale : c'est premièrement sur le plan des cultures que cette transformation aura lieu. Comment placer alors la ville durable entre les mains des architectes et des urbanistes, étant donné qu'ils ne sont pas ses " pères fondateurs »?

Certaines recherches récentes ont enquêté sur les antécédents historiques de la notion de ville durable dans le domaine de l'architecture et de l'urbanisme, soit pour en saisir les dangers, soit pour la replacer dans le débat disciplinaire. Pour certains ${ }^{12}$, I'insertion de cette notion dans une perspective historique est un moyen pour parvenir à une vision critique de l'usage rhétorique qu'on en fait parfois. Cette opération se déroule à travers la mise en rapport de la ville durable avec d'autres impératifs d'action sur le rapport nature/ ville qui ont jalonné I'histoire urbaine récente (décor urbain, hygiénisme, écologie urbaine). Pour d'autres ${ }^{13}$, l'insertion dans une perspective historique est un moyen pour développer la composante culturelle du concept par rapport à ses composantes économiques, sociales et environnementales : la ville durable se poserait en continuité avec l'urbanisme culturaliste. Frederick Law Olmsted, Ebenez Howard et Patrick Geddes sont présentés comme inspirateurs de la ville durable, puisqu'ils proposent la restructuration de la ville à travers la "nature » et indiquent l'échelle territoriale comme pertinente pour développer la réflexion sur I'urbain ${ }^{14}$.
9. Nous croyons que ce type de réflexion a animé le débat sur le statut de

l'architecture et sur son autonomie en tant que production intellectuelle depuis « les évènements de $1968 »$. Voir Jean-Louis Violeau, Les architectes et Mai 68, Paris, Éditions Recherches, 2005.

10. «Ses auteurs ne sont plus des architectes, des urbanistes, [...] mais surtout des experts scientifiques, des politiques, des organismes internationaux, des associations écologistes nationales et internationales »: Albert Lévy, « La "ville durable". Paradoxes et limites d'une doctrine d'urbanisme émergente. Le cas Seine Arche », Esprit, décembre 2009, p. 143

11. Cyria Emelianoff, "La ville durable: I'hypothèse d'un tournant urbanistique en Europe », L'information géographique $n^{\circ} 3$, 2007, p. 48-65.
12. Sophie Descat et al. (dir.), La ville durable au risque de l'histoire, Paris, Jean-Michel Place, 2006

13. Ingrid Ernst (dir.), Cultures urbaines et développement durable, Paris, ministère de l'Aménagement du territoire et de I'Environnement, 2002.

14. C. Emelianoff, La ville durable : une culture en gestation, dans I. Ernst (dir.), op. cit. note 13, p. $43-64$ 
Sur les fondements et les modalités de cette mise en perspective de la ville durable, plusieurs questions restent ouvertes. Le questionnement historique peut-il contribuer à un avancement de la définition de la ville durable ? Quels sont les critères et les méthodes pour effectuer une mise en perspective historique pertinente de la ville durable ? Nous croyons que la question de l'insertion de la ville durable dans une perspective historique est une tâche nécessaire mais complexe et que ses antécédents doivent être choisis avec précaution. En effet, un processus de franchissement progressif des principes du fonctionnalisme est repérable en France à partir des années 1970, donc bien avant la diffusion du développement durable ${ }^{15}$. II reste à clarifier si et comment la ville durable peut s'inscrire dans cette perspective. Enfin, on peut se demander si et dans quelle mesure la recherche des antécédents de la ville durable n'est pas plutôt une tentative pour la légitimer au sein de la communauté des urbanistes.

\section{Du modèle aux espaces : instrumentalisation}

Suite au questionnement épistémologique lié à la ville durable, un " retour à la réalité » de cette notion s'impose. L'instrumentalisation de la ville durable dans les domaines de l'architecture et de l'urbanisme nous oblige à aborder la question des modèles. Comme la ville durable n'est pas porteuse d'un modèle spatial précis mais qu'elle est construite à partir de déclinaisons locales et arrangements successifs, comment peut-elle devenir un modèle ? Si on ne peut pas parler de modèle spatial, de quel type de modèle parlons-nous? S'agit-il d'une démarche opérationnelle, ou d'une approche culturelle ? De fait, certaines recherchesactions démontrent qu'une certaine reproductibilité est possible, ou du moins qu'elle est envisagée par les acteurs urbains ${ }^{16}$

Par exemple, la comparaison entre la Charte d'Aalborg ${ }^{17}$, considérée comme manifeste de la doctrine ${ }^{18}$ de la ville durable, et la Charte d'Athènes, peut contribuer à articuler la réflexion sur les modèles. La Charte d'Aalborg a été définie comme " anti-Charte $d^{\prime}$ Athènes ${ }^{19}$ ", puisque si toutes les deux s'interrogent sur le rapport entre ville et nature, elles en donnent toutefois des interprétations opposées. La Charte d'Athènes propose un modèle spatial précis dérivé de la vision fonctionnaliste de la ville et d'une vision essentiellement positive de la société développée autour de la notion de progrès, tandis que la Charte d'Aalborg, fondée sur une démarche globale, ne propose pas un modèle spatial de référence et prend conscience de la finitude de l'espace et des ressources. En revanche, l'élément commun de ces deux visions n'a guère été souligné : toutes les deux font référence à l'idée de l'urbanisme comme pratique technique.

L'instrumentalisation de la ville durable implique son insertion dans le contexte de l'architecture et l'urbanisme, en tant que disciplines et en tant qu'ensemble d'actions. La prise en compte de la dimension spatiale est centrale, puisque c'est sur ce plan qu'a lieu la représentation propre à ces disciplines. D'ailleurs, même si le concept de ville durable attend toujours une définition partagée, la question des conséquences spatiales $^{20}$ de la durabilité urbaine aux différentes échelles se pose avec urgence.
15. Voir Jean-Louis Cohen, «La coupure entre architectes et intellectuels. Les enseignements de l'italophilie », In Extenso $n^{\circ} 1$, Paris, École d'architecture de Paris-Villemin, 1984 ; Fausto Carmelo Nigrelli, Percorsi del progetto urbano in Francia e in Italia 1960-1997, Rome, Officina Edizioni, 1999

16. Nous citons à ce propos le programme de recherche urbaine, POPSU (Plate-forme d'observation des projets et stratégies urbaines) lancé en 2003 par le GIP EPAU devenu GIP AIGPPAU. II est piloté par le PUCA (Plan Urbanisme, Construction, Architecture).

17. "Charte des villes européennes pour la durabilité ", adoptée par les participants à la Conférence européenne sur les villes durables qui s'est tenue à Aalborg, Danemark, le 27 mai 1994
18. Sur le concept de doctrine, voir Jean-Pierre Epron, L'architecture et la règle. Essai d'une théorie des doctrines architecturales, Bruxelles-Liège, Pierre Mardaga, 1981.

19. A. Lévy, op. cit. note 10. C. Emelianoff, "Les villes européennes face au développement durable : une floraison $d^{\prime}$ initiatives sur le fond de désengagement politique ", Les Cahiers du PROSES $n^{\circ} 8$, janvier-février 2004. 
La question de l'espace urbain dans les discours des architectes et des urbanistes s'est placée au fil du temps entre deux extrêmes. Le premier réduit la question aux catégories de l'espace physique; le second, influencé par les sciences humaines, envisage la dimension physique de l'espace comme support (indifférent?) de phénomènes sociaux. Entre ces deux extrêmes, nous soulignons l'intérêt du projet urbain, processus qui considère comme centrale la question de la transformation de l'espace et qui s'est ouvert au cours des années 1980 et surtout 1990 aux questions liées à la prise en compte de la complexité de la ville contemporaine. Nous croyons qu'une mise en articulation du projet urbain et de la ville durable - avec les continuités et les ruptures qu'elle présuppose - est nécessaire. Cette opération pourrait conduire à un avancement de la notion de ville durable dans le champ de la recherche urbaine, surtout concernant ses aspects opératifs et sa territorialisation.

\section{Conclusion}

Nous avons essayé d'explorer dans ce texte la portée et les limites de la notion de ville durable, dans l'intention de comprendre sa position dans la recherche urbaine. Une des questions qui reste ouverte est celle-ci : quelle posture intellectuelle adopter, en tant qu'architecte et urbaniste, face à la ville durable? Nous faisons référence ici au champ d'action de la recherche dessiné au début de cette réflexion par quatre personnages pittoresques.

La position des "prétoriens " - qui insistent sur I'autonomie disciplinaire - semble être hors contexte face à un concept polysémique tel que la ville durable.
Celle des " aventuriers » est peut être l'une des postures les plus communes : beaucoup parlent de ville durable, mais très peu sont ceux qui en cherchent une déclinaison qui soit reconductible dans leur discipline d'origine. Les " notaires ", qui voient la recherche comme un enregistrement neutre de la réalité, risquent de ne pas trouver grande chose à enregistrer, puisque la ville durable n'est pas un objet empirique. Les " ventriloques" (il y en a beaucoup), qui croient que la réalité dérive seulement de leur propre activité de recherche, risquent de se retrouver avec un objet bien défini et scientifiquement fonctionnel, mais qui ne répond pas aux problèmes réels de la ville (par exemple la question de la démocratie), surtout à cause du fait que la ville durable est issue d'une représentation politique.

Ces postures scientifiques caricaturales qui nous aident malgré tout à réfléchir sur les directions actuelles de la recherche sur la ville durable mettraient-elles en jeu un cinquième personnage : l'architecte-urbaniste intellectuel qui, comme le soulignait Umberto Eco ${ }^{21}$, essaie de dialectiser à travers une démarche à la fois épistémologique et opérationnelle les questions et les enjeux de son temps?

20. Qui n'est qu'une partie de la question.

Cependant elle reste centrale dans la

réflexion et la pratique de l'architecture et de l'urbanisme.

21. Umberto Eco, Préface, dans Vittorio Gregotti, Le territoire de l'architecture, Paris, L’Équerre, 1982 [1éd. 1966]. 